Tuberkulose. Seit 1894 leidet Patientin an Pharyngitis. Im September vorigen Jahres erkrankte sie an doppelseitiger Lungen-Brustfellentzündung und Diphtherie. Seit dieser Zeit klagt sie über Seitenstechen und zeitweisen Husten mit Auswurf, was immer heftiger geworden ist.

Lungenbefund: Links vorn und hinten oben leichte Dämpfung mit kleinblasigen Rasselgeräuschen, unterhalb der Scapula Reibegeräusche. Rechts hinten oben Schallverkürzung, wo Inspirium verschärft und Exspirium verlängert ist. Im Sputum Tuberkelbazillen nicht nachzuweisen. (Siehe vorstehende Tabelle.)

Fall 2. Marie D., Plätterin, 30 Jahre alt, aufgenommen am 30. April 1902.

Ein Bruder der Patientin ist vor sechs Jahren an der Schwindsucht gestorben. Eine Schwester ist seit etwa 17 Wochen lungenkrank. Patientin hat mit ihrem Bruder zusammengewohnt und ihn gepflegt. Seit Dezember leidet Patientin an Husten, verbunden mit ziemlich starkem Auswurf nnd Nachtschweissen, wozu sich auch zeit

III. Aus dem Institut für Infektionskrankheiten in Berlin. (Direktor: Geh. Med.-Rath Prof. Dr. Koch.)

\section{Die Vortheile der combinirten Behandlung bei der chronischen Tuberkulose.}

\section{Von Dr. J. Mitulesen in Bukarest.}

(Schluss aus No. 21.)

In folgendem theile ich einige kurze Beobachtungen, von der betreffenden Stoffwechselbilanz begleitet, mit, welche das oben Erläuterte bestätigen und die dringende Anwendung der diätetospezifischen Behandlung in der Tuberkulose als allein zu einer N-Retention im Organismus befähigt, erweisen sollen:

Fall 1. Auguste Schinkat, Wäscherin, 38 Jahre alt, aufgenommen am 23. Mai 1902. Die Anamnese bietet keine Anhaltspunkte für die

\begin{tabular}{|c|c|c|c|c|c|c|c|c|c|c|}
\hline \multirow{2}{*}{\multicolumn{2}{|c|}{$\begin{array}{c}\text { Ein- } \\
\text { nahme }\end{array}$}} & \multicolumn{6}{|c|}{ Ausgabe } & \multirow{2}{*}{\multicolumn{2}{|c|}{ Bilanz }} & \multirow{3}{*}{$\begin{array}{c}\text { Tem- } \\
\text { peratur }\end{array}$} \\
\hline & & \multicolumn{2}{|c|}{ Koth } & \multicolumn{4}{|c|}{ Urin } & & & \\
\hline $\mathbf{N}$ & $\mathrm{Ph}$ & $\mathrm{N}$ & $\mathrm{Ph}$ & $\begin{array}{l}\mathrm{Da}- \\
\text { tum }\end{array}$ & $\begin{array}{c}\text { Men- } \\
\text { ge }\end{array}$ & $\mathrm{N}$ & $\mathrm{Ph}$ & $\mathrm{N}$ & $\mathrm{Ph}$ & \\
\hline $13,2 \overline{5}$ & 3,76 & $\mid \begin{array}{r}1,13 \mid \\
\text { (D. }\end{array}$ & $\begin{array}{l}1.24 \\
\text { t.) }\end{array}$ & $\begin{array}{l}\text { Mai } \\
28 .\end{array}$ & 1100 & 11,63 & 2,35 & $+0,42$ & +0.17 & $36,2-36,8$ \\
\hline & & & & 29. & $\frac{1200}{1015}$ & 11,98 & 2,43 & $+0,14$ & $+0,09$ & $36,3-36,9$ \\
\hline & & & & 30. & $\frac{1250}{1015}$ & 11,51 & 2,15 & $+0,69$ & $+0,37$ & $36,3-36,9$ \\
\hline & & & & 31. & 1350 & 11,06 & 1,98 & $+1,06$ & $+0,54$ & $36,5-37$ \\
\hline 17,25 & 3,782 & 2,48 & 1,34 & $\begin{array}{l}\text { Juni } \\
1 .\end{array}$ & $\begin{array}{l}1015 \\
1200\end{array}$ & 13,86 & 2,246 & $+0,91$ & +0.196 & $36,4-37$ \\
\hline $\begin{array}{r}\text { Von } d \\
(4 \mathrm{~g}\end{array}$ & $\begin{array}{l}\text { lenen } \\
N\end{array}$ & (D) & & $?$ & $\begin{array}{l}101 \overline{8} \\
1200\end{array}$ & & & 19 & +0289 & $364-369$ \\
\hline 0,022 & $\mathrm{Ph})$ & & & 2. & $\frac{1900}{1016}$ & 10,60 & $\mid 2,100$ & $+1,12$ & $+-0,209$ & $00,4--0,9$ \\
\hline $\begin{array}{l}\text { von } \\
\text { Robo }\end{array}$ & $\begin{array}{l}25 \mathrm{~g} \\
2 \mathrm{rat}\end{array}$ & & & 3. & $\frac{1200}{1017}$ & 12,95 & 1,95 & $+1,72$ & $+0,492$ & $36,5-37$ \\
\hline & & & & 4. & $\frac{1150}{1017}$ & 12,86 & 1,782 & $+1,92$ & $+0,66$ & $36,6-37$ \\
\hline & & & & 5. & $\frac{1250}{1018}$ & 13,15 & 1,951 & $+1,62$ & $+0,491$ & $36,5-37$ \\
\hline & & & & 6. & $\frac{1200}{1018}$ & 13,68 & 2,14 & $+1,09$ & $+0,302$ & $36,6--37$ \\
\hline $\begin{array}{r}21,25 \\
(8 \mathrm{~g}\end{array}$ & $\frac{3.804}{\mathrm{~N}}$ & $\begin{array}{r}5,62 \\
\text { (D. }\end{array}$ & $\begin{array}{l}1,28 \\
t .)\end{array}$ & 7. & 1150 & 13,91 & 2,48 & $+1,72$ & $+0,044$ & $36,5-37,1$ \\
\hline $\begin{array}{r}0,0435 \\
\text { in } 5\end{array}$ & Ph) & & & 8. & $\frac{1200}{1090}$ & 14,45 & 2,253 & $+1,18$ & $+0,271$ & $36,8-37,4$ \\
\hline Robo & & & & 9. & $\begin{array}{l}1020 \\
1150 \\
\end{array}$ & 13,88 & 2,153 & $+1,75$ & $+0,371$ & $36,3-37$ \\
\hline & & & & 10. & $\begin{array}{l}1019 \\
1250 \\
1020\end{array}$ & 13,14 & 2,38 & $+2,49$ & $+0,164$ & $36,5-37$ \\
\hline & & & & 11. & $\frac{1150}{1019}$ & 12,85 & 2,153 & $+2,78$ & $+0,371$ & $36,5-37,2$ \\
\hline & & & & 12. & $\frac{1250}{1090}$ & 12,71 & 2,486 & $+2,02$ & $+0,068$ & $36,8-37,1$ \\
\hline & & & & 13. & $\left|\frac{1200}{1020}\right|$ & 12,986 & 2,354 & $+2,634$ & $+0,170$ & $36,9-37,1$ \\
\hline
\end{tabular}

1) ibidem 1901, No $11,{ }^{2}$ ) ibidem 1903 , No. 5, Tabelle II.

3) Während sich dieser Aufsatz im Druck befand, erschien eine Arbeit von Wassermann und Schütze (ibidem 1903, No. 11), in der sie besonders betonen, dass diese von Wassermann erfundene Differenzirungsmethode nur eine "Artreaktion“, $d . h$. eine Reaktion auf eine Spezies ergeben könnte; ich glaube, durch meine experimentellen Versuche und theoretischen Erwägungen bewiesen zu haben, dass neben der "Artreaktion" in gewissen Fällen auch eine Reaktion bestimmter Organbestandtheile, soweit dieselben ihnen spezifische Bestandtheile enthalten, auszulösen ist. weise Brustschmerzen gesellen. Vor vier Monaten hatte sie zweimal blutigen Auswurf. Patientin hat schlaffe Muskulatur und geringes Fettpolster. Die Schleimhäute sind etwas blass. - An der rechten Spitze Schallabschwächung mit bronchialem Athmen und kleinen blasigen Rasselgeräuschen. Links oben vorn rauhes Inspirium, Giemen und Pfeifen, hinten kleinblasige Rasselgeräusche, unten Lederknarren. Im Sputum sind Tuberkelbazillen vorhanden.

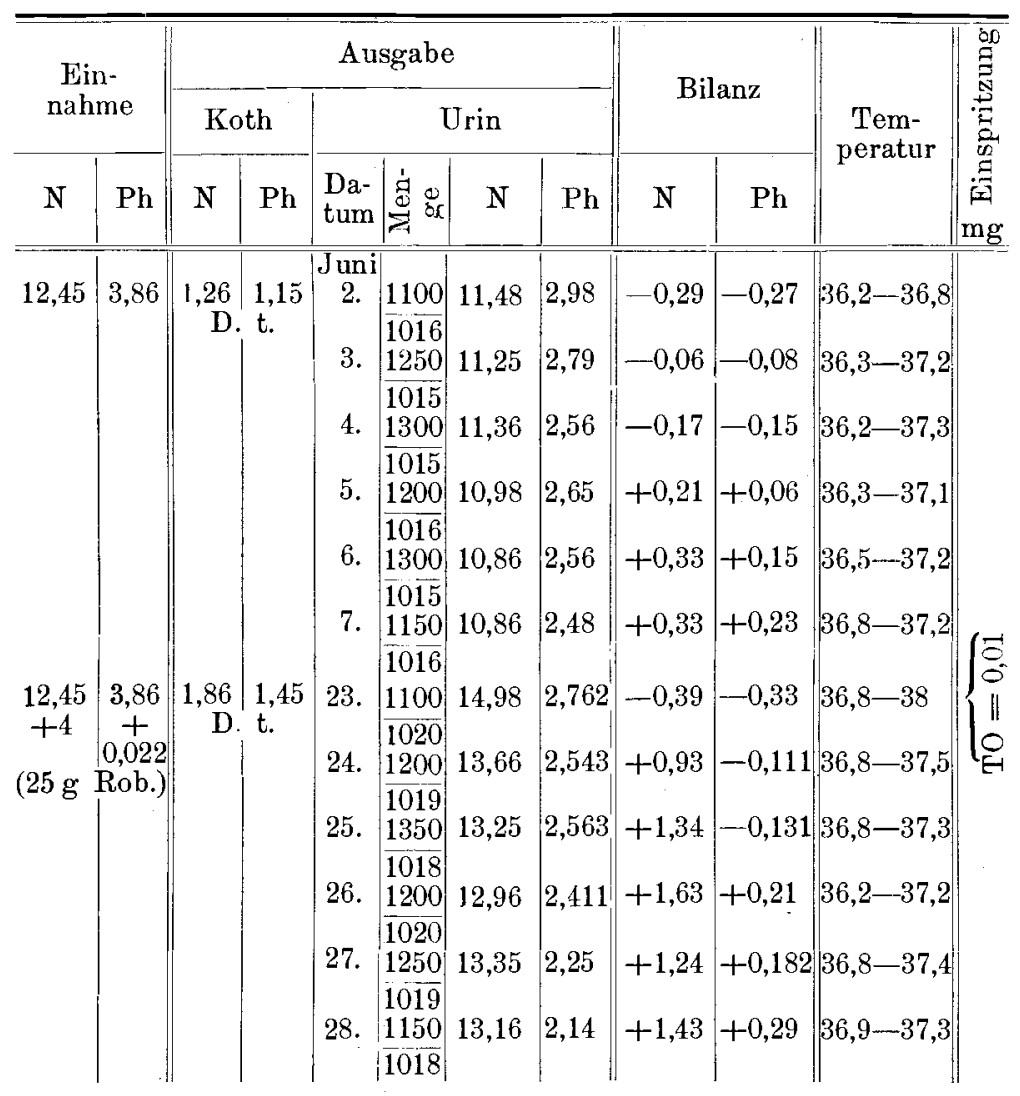

Fall 3. A. Babel, Näherin, 18 Jahre alt, aufgenommen am 22. April 1902.

Es lässt sich von Tuberkulose anamnestisch nichts nachweisen. Patientin hat ihr Leben unter ungünstigen Umständen zurückgelegt. Im August v. J. trat zunächst heftiger, trockener Husten auf, dem sich sechs Wochen später Auswurf, sowie Nachtschweisse zugesellten. Nach ärztlicher Behandlung wurden die Beschwerden gelinder. Anfang: März stellten sich obige Erscheinungen wieder ein, diesmal mit Schmerzen in der linken Seite. Darauf machten sich Mattigkeit, Appetit- und Schlaflosigkeit bemerkbar. - Ueber der linken Spitze Schallabschwächung, Bronchialathmen und kleinblasige Rasselgeräusche. Rechts vereinzelte Rasselgeräusche. Im Sputum Tuberkelbazillen.

\begin{tabular}{|c|c|c|c|c|c|c|c|c|c|c|c|}
\hline \multirow{2}{*}{\multicolumn{2}{|c|}{$\begin{array}{c}\text { Ein- } \\
\text { nahme }\end{array}$}} & \multicolumn{6}{|c|}{ Ausgabe } & \multirow{2}{*}{\multicolumn{2}{|c|}{ Bilanz }} & \multirow{3}{*}{$\begin{array}{c}\text { Tem- } \\
\text { peratur }\end{array}$} & \multirow{3}{*}{ 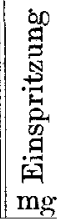 } \\
\hline & & \multicolumn{2}{|c|}{ Koth } & \multicolumn{4}{|c|}{ Urin } & & & & \\
\hline $\mathrm{N}$ & $\mathrm{Ph}$ & $\mathrm{N}$ & $\mathrm{Ph}$ & $\begin{array}{l}\text { Da- } \\
\text { tum }\end{array}$ & $\sum_{0}^{1}$ & $\mathrm{~N}$ & $\mathrm{Ph}$ & $\mathrm{N}$ & $\mathrm{Ph}$ & & \\
\hline 13,25 & 3,76 & 年, & $\begin{array}{l}1,22 \\
t .\end{array}$ & $\begin{array}{c}\text { Mai } \\
7 . \\
8 . \\
9 .\end{array}$ & $\left|\begin{array}{|}1000 \\
1016 \\
1200 \\
1015 \\
1150 \\
1015\end{array}\right|$ & $\begin{array}{l}12,25 \\
12,05 \\
11,83\end{array}$ & $\begin{array}{l}2,86 \\
2,68 \\
2,25\end{array}$ & $\begin{array}{l}-0,34 \\
-0,14 \\
+0,08\end{array}$ & $\begin{array}{l}-0,32 \\
-0,14 \\
+0,29\end{array}$ & $\left|\begin{array}{l}36,9-37 \\
36,9-37,1 \\
36,7-37\end{array}\right|$ & \\
\hline
\end{tabular}




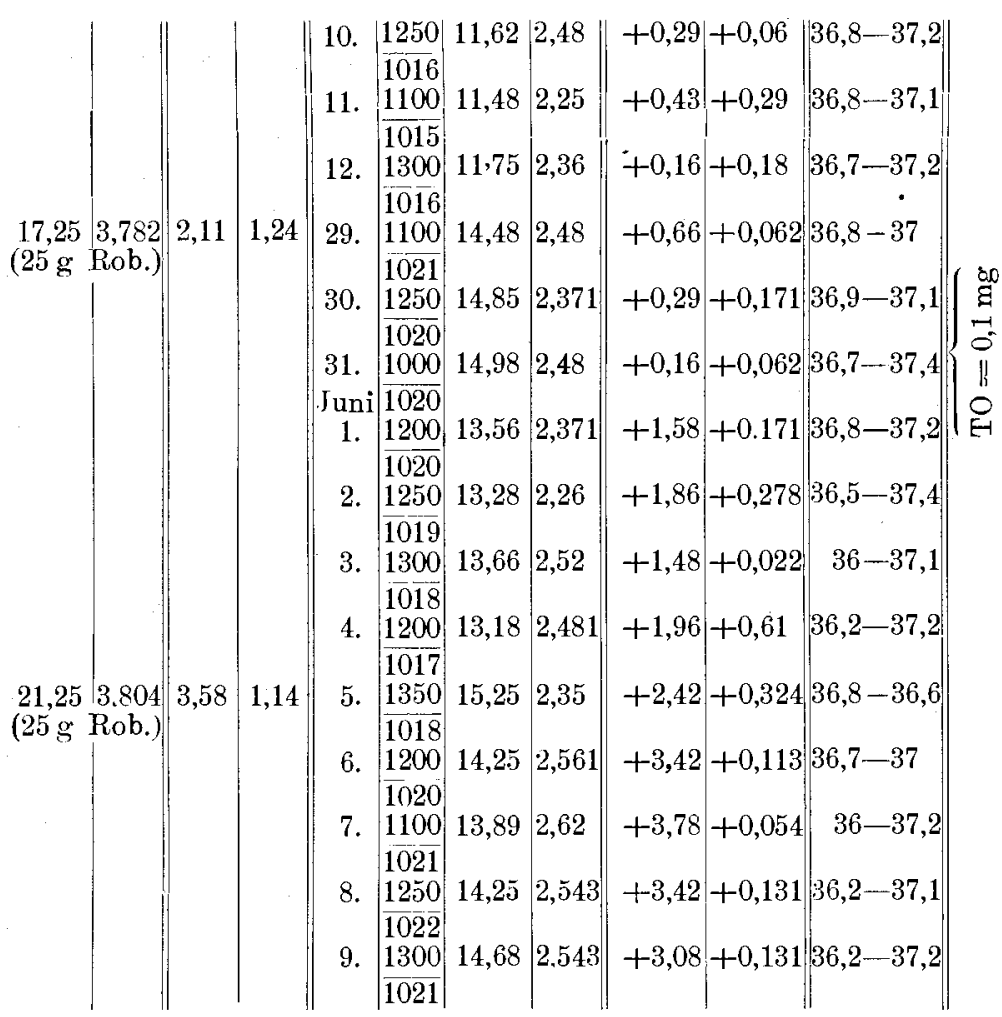

Fall 4. H. Hoffmann, Schneiderin, 34 Jahre alt, aufgenommen am 19. April 1902.

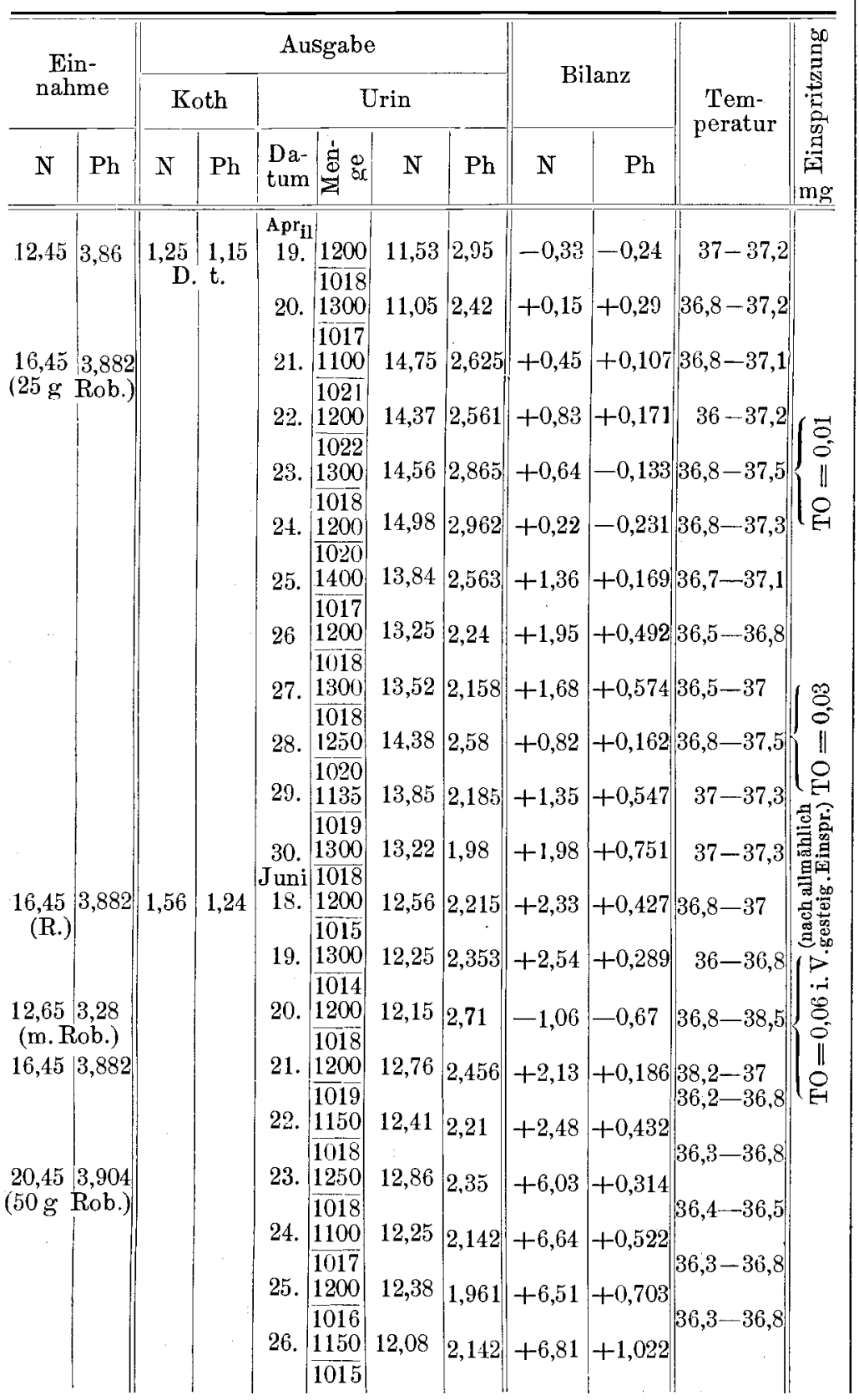

Mutter der Patientin ist zuckerkrank; ihr Vater hustet seit letztem Winter und klagt über Luftmangel. Sie lebt in günstigen hygienischen Verhältnissen. Vor einem Jahre hatte sie Brustfellentzündung. Seit September klagt sie über Schmerzen und Stiche in der linken Brustund Rückenseite, sowie über grosse Mattigkeit, Husten und Auswurf, die seitdem bestehen blieben.

Vor acht Tagen trat blutiger Auswurf auf, welcher drei Tage andauerte.

Lungenbefund: Links vorn oben leichte Dämpfung, verschärftes Inspirium, Giemen und kleinblasige Rasselgeräusche, hinten oben bronchiales Athmen, unten Lederknarren. Im Sputum Tuberkelbazillen.

Aus diesen Tabellen ist ersichtlich, dass im ersten, nur hygieno-diätetisch behandelten Falle bei vermehrter Stickstoffzufuhr, trotz normaler Darmthätigkeit, die Vermehrung des $\mathrm{N}$ - und $\mathrm{Ph}$-Ansatzes nur eine sehr langsame und allmähliche war, die Verluste durch den Darm verhältnissmässig gross waren. Bei den anderen drei Fällen waren genau conforme Verhältnisse zu constatiren, solange bis die Tuberkulinbehandlung einsetzte; von diesem Momente an wurden die Eiweissverluste durch die Fäces zwar auch vermehrt, aber lange nicht so erheblich, wie in dem ersten Falle, während $\mathrm{N}$ - und Ph-Ansatz eine ganz bedeutende Steigerung aufwiesen.

Ein grosser Proteïnansatz ist nur nach mehreren Einspritzungen mit Tuberkulin möglich. Die Ursache dafür beruht darin, dass die Zellen sich in einem bedeutenden Erregungszustande befinden und folglich, da ihre Lebensfähigkeit vergrössert ist, die Assimilationsmöglichkeit um bedeutendes gestiegen ist. 